\title{
Transcatheter Closure of Double Ventricular Rupture in a Patient Complicating Acute Myocardial Infarction
}

\author{
Chin Chang Cheng*1, Tse Hsuan ${ }^{2}$ and Yang ${ }^{2}$ \\ ${ }^{1}$ Department of Cardiovascular Center, National Yang-Ming University, Taiwan \\ ${ }^{2}$ Department of Cardiovascular Center, Medical University of Silesia, Kaohsiung, Taiwan
}

Received: March 19, 2018; Published: April 06, 2018

*Corresponding author: Chin Chang Cheng, Department of Cardiovascular Center, National Yang-Ming University, Taipei, Taiwan, Tel: 886-7-3468278;

Email: shyane0707@gmail.com

\begin{abstract}
Ventricular septal rupture (VSR) is a fatal complication of acute myocardial infarction. The incidence of VSR is increasingly rare in the percutaneous coronary intervention era but mortality rate remains high. The key to management of this critical condition is an aggressive approach to hemodynamic stabilization and surgical closure of the rupture. In some patients, transcatheter closure may be considered as an alternative way of treatment. There were several case series demonstrated that percutaneous closure as a bridge to surgery improves outcome of patient who has VSR with hemodynamic ally unstable.Wepresent a case who received transcatheter closure of double ventricular rupture with pseudoaneursym after initial surgical repairment of post infarction ventricular septal rupture.
\end{abstract}

Keywords: Ventricular Septal Rupture; Acute Myocardial Infarction; Mechanical Complications; Transcatheter Closure

Abbreviations: VSR: Ventricular Septal Rupture; AMI: Acute Myocardial Infarction; PCI: Percutaneous Coronary Intervention; LAD: Left Anterior Descending Artery; LLSB: Left Lower Sternal Border; CT: Computer Tomography; CAG: Coronary Angiogram; LV: Left Ventricle; RV: Right Ventricle; Post-Mi VSR: Post Infarction Ventricular Septal Rupture; QP: Pulmonary Blood Flow Ratio; QS: Systemic Blood Flow Ratiov

\section{Introduction}

Ventricular septal rupture (VSR) follow by acute myocardial infarction (AMI) is a rare but fatal complication. Due to the progressive development and availability of reperfusion therapy, the incidence of VSR in last decades has declined between 0.17 and $0.31 \%[1,2]$. Despite the improvements in early diagnosis and treatment of both AMI and VSR, the mortality rate from VSR remains extremely high ranging from 45-80\% [1-4]. Surgical ventricular septal rupture repairment has been the golden standard of treatment, but challenging operation associated with high early mortality [5]. Since the structure cardiac intervention has evolved in past decades, the percutaneous ventricular septal rupture closure became the other option for patients with significant risk for surgical repair. Moreover, there have been several case series demonstrated that percutaneous closure as a bridge to surgery improves outcome of patient who has VSR with hemodynamic ally unstable [6-9].We present a case who received transcatheter closure of double ventricular rupture with pseudoaneursym after initial surgical repairment of post infarction ventricular septal rupture.

\section{Case Report}

A 68 years old lady has medical history of hypertension, Diabetes Mellitus with nephropathy under maintenance hem dialysis for decades. She had acute myocardial infarction underwentpercutaneous coronary intervention (PCI) with drugeluting stent placement over proximal part left anterior descending artery (LAD) in 2015. Three days after the procedure, she experienced worsen exceptional dyspnea and grade III holosystolic heart murmur over left lower sternal border (LLSB) was auscultator, transthoracic echocardiogram revealed post in fractional apical VSR (Figure 1). She received surgical repairment with synthetic substitute three weeks after the AMI. The patient had visited cardiology outpatient department regularly afterward. After several months, she started to complain about dizziness and chest tightness with progress exceptional dyspnea. A grade III holosystolic heart murmur was auscultator over LLSB and apex area. Chest computer tomography (CT) revealed a new pseudoaneursym formation over left ventricular anterior wall below the surgical substitute (Figure 2). The following transesophageal echocardiogram revealed interventricular shunt between left ventricular (LV), 
right ventricular (RV), and pseudoaneursym over apex area. The pseudoaneursym had raged edge, narrow neck which was high risk for perforation. Furthermore, the coronary angiography (CAG) revealed diffuse atherosclerosis over distal portion of LAD with dynamic compression by the pseudoaneursym (Figure 2). After thorough evaluation of the heart team, percutaneous transcatheter closure of VSR and pseudoaneursym were preceded about 6 months after initial myocardial infarction episode. The percutaneous closure of double ventricular rupture was performed twice aim to reduction in residual left-to-right shunting (QP: QS). The first intervention, $16 \mathrm{~mm}$ Amplatzer muscular VSD occluder was deployed over one of fenestrated VSR of LV pseudoaneursym. The second intervention, we choose the $6 \mathrm{~mm}$ Amplatzer septal occluder according to the morphology which we measured by sizing balloon (Figure 3). Three months after VSR occluder intervention, her clinical symptoms improved, and the following chest CT showed no significant progression of pseudoaneursym size, the transthoracic echocardiography could revealed no residual shunt.

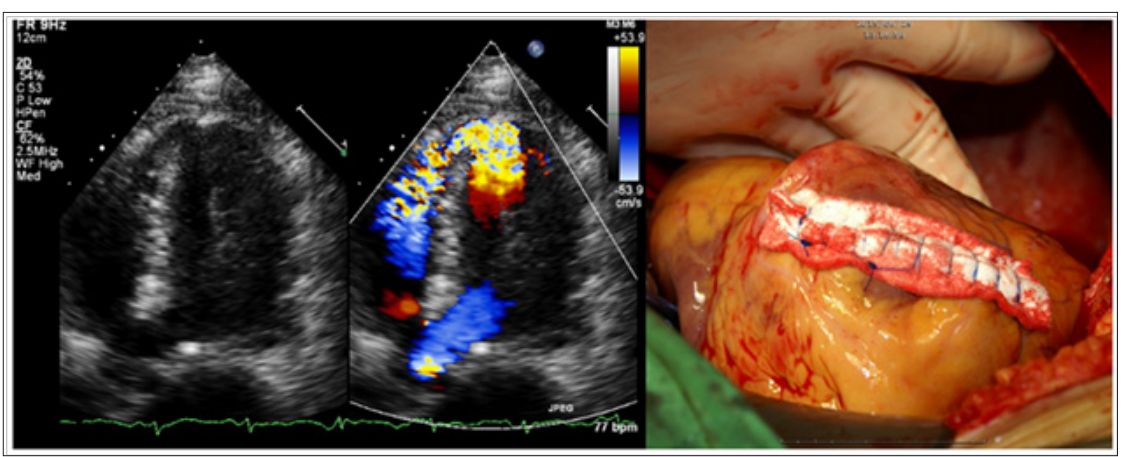

Figure 1: Postinfarction VSR \& surgical repairmen.

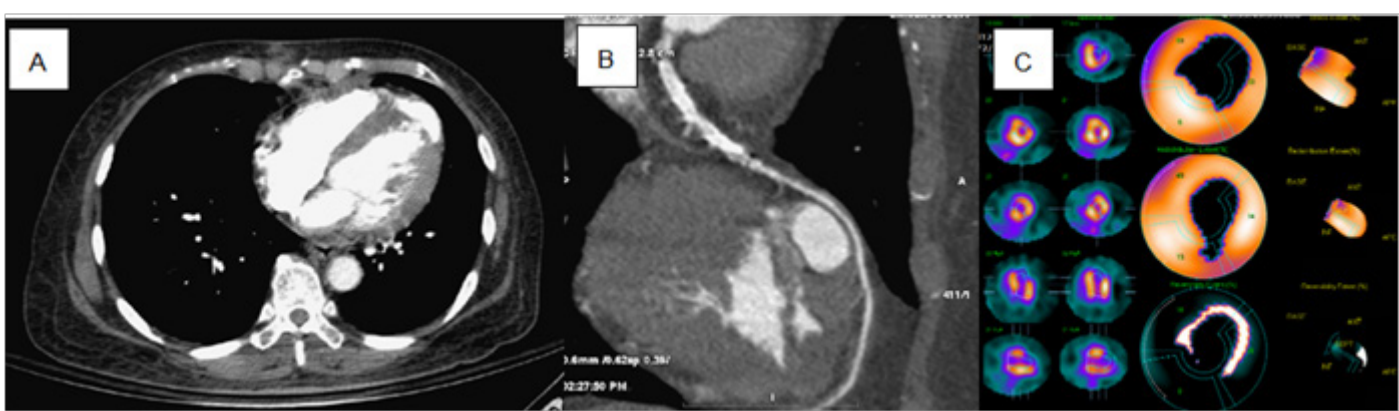

Figure 2: A. Chest CT: Psuroaneurysm; B. CAG: Dynsmic compression of LAD; C. Thallium scan: ischemia changes over anterolateral territories.

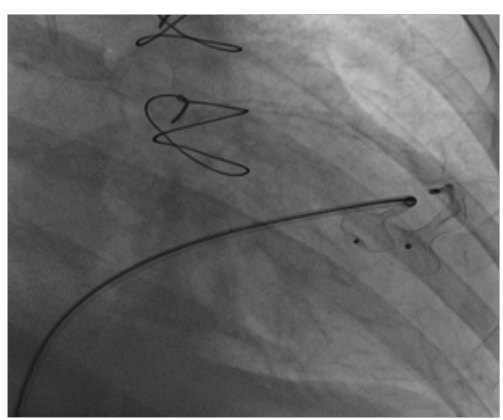

Figure 3: Amplatzer muscular VSD occlude \& Amplatzer septal occlude.

\section{Discussion}

VSRis a serious complication of acute myocardial infarction. Early diagnosis and detection of VSR is the crucial element for survival. All patients who develop hemodynamic compromise during AMI should be rapidly examined for the characteristic, systolic murmur over the pericardium. The echocardiogram Doppler techniques can be used to define the site and size of septal rupture, left and right ventricular function, estimated right ventricular systolic pressure, and the left-to-right shunt. Pulmonary-artery catheterization, Left ventriculography, and transesophageal echocardiography can also be helpful. In our case, the suspicious of post infarction VSR and recurrent LV pseudoaneursym were made by holosystolic murmur initially. Accurate timing of surgical intervention for post infarction VSR who is hemodynamic ally stable remains unclear. It is clear from the published surgical series that operative mortality is reduced by delaying surgery $[10,11]$. Others trial also demonstrate that although surgical mortality remains very high, non-surgical mortality is certainly higher [2].

Despite current guideline, some consensus recommends that timing of surgery should be considered according to the hemodynamic ally condition, and size of VSR shunt [12]. In patients who are able to maintain hemodynamic stability with and without medical support, a delayed surgical approach with use of circulatory support is the preferred method for VSR repair [13]. In our case, the surgical repairment was performed three weeks after acute myocardial infarction, but the timing seems not sufficient enough for fully recovery of infarct related muscle. From the technical point of view, attempting device placement 
requires a thorough understanding of the size, shape, and borders. Complete image examinations, including ECG-gated computed tomography angiography and echocardiography (transthoracic and transesophageal), left ventrculography, coronary angiography are crucial in management of ventricular septal rupture, especially in whom accompanied with pseudoaneursym formation.

\section{Conclusion}

We concluded that the post infarction ventricular septal rupture (post-MI VSR) of apical septum was difficult to diagnose on transthoracic echocardiography, and new-onset holosystolic murmur is an important clue. Although there is no consensus on timing of surgical intervention of the post-MI VSR, surgical intervention may be deferred as long as possible if hemodynamic stable. Complete image examinations, including ECG-gated computed tomography angiography and echocardiography (transthoracic and transesophageal) are important for transacted closure of VSR. Transcatheter closure is feasible in patients with residual VSR or pseudoaneursym after surgical repair of post-MI VSR.

\section{References}

1. Moreyra AE, Huang MS, Wilson AC, Deng Y, Cosgrove NM, et al. (2010) Trends in incidence and mortality rates of ventricular septal rupture during acute myocardial infarction. Am J Cardiol 106: 1095-1100.

2. Crenshaw BS, Granger CB, Birnbaum Y, Pieper KS, Morris DC, et al (2000) Risk factors, angiographic patterns, and outcomes in patients with ventricular septal defect complicating acute myocardial infarction. GUSTO-I (Global Utilization of Streptokinase and TPA for Occluded Coronary Arteries) Trial Investigators. Circulation 101: 27-32.

3. Lopez Sendon J, Gurfinkel EP, Lopez de Sa E, Agnelli G, Gore JM, et al. (2010) Factors related to heart rupture in acute coronary syndromes in the Global Registry of Acute Coronary Events. Eur Heart J 31: 14491456 .
4. French JK, Hellkamp AS, Armstrong PW, Cohen E, Kleiman NS, et al. (2010) Mechanical complications after percutaneous coronary intervention in ST-elevation myocardial infarction (from APEX-AMI). Am J Cardiol 105: 59-63.

5. Arnaoutakis GJ, Zhao Y, George TJ, Sciortino CM, McCarthy PM (2012) Surgical repair of ventricular septal defect after myocardial infarction: outcomes from the Society of Thoracic Surgeons National Database. Ann Thoracic Surg 94: 436-443.

6. Thiele H, Kaulfersch C, Daehnert I, Schoenauer M, Eitel I, et al. (2009) Immediate primary transcatheter closure of postinfarction ventricular septal defects. Eur Heart J 30: 81-88.

7. Attia R, Blauth C (2010) Which patients might be suitable for a septal occluder device closure of postinfarction ventricular septal rupture rather than immediate surgery? Interact Cardiovasc Thorac Surg 11: 626-629.

8. Assenza GE, McElhinney DB, Valente AM, Pearson DD, Volpe M, et al. (2013) Transcatheter closure of post-myocardial infarction ventricular septal rupture. Circ Cardiovasc Intervent 6: 59-67.

9. Maltais S, Ibrahim R, Basmadjian AJ, Carrier M, Bouchard D, et al. Postinfarction ventricular septal defects:towards a new treatment algorithm? Ann Thoracic Surg 87: 687-692.

10. Arnaoutakis GJ, Zhao Y, George TJ, Sciortino CM, McCarthy PM, et al. (2012) surgical repair of ventricular septal defect after myocardial infarction: outcomes from the Society of Thoracic Surgeons National Database. Ann Thorac Surg 94: 436-443.

11. Deja MA, Szostek J, Widenka K, Szafron B, Spyt TJ, et al. (2000) Post infarction ventricular septal defect: can we do better? Eur J Cardiothorac Surg 18: 194-201.

12. Brandon M Jones, Samir R Kapadia, Nicholas G, Michale Robich, Amar Krishnaswamy et al. (2014) Ventricular septal rupture complicating acute myocardial infarction: a contemporary review. European Heart Journal 35: 2060-2068.

13. Jared J Liebelt, Yuanquan Yang, Joseph J DeRose, Cynthia C Taub, Jared J Liebelt, et al. (2016) Ventricular septal rupture complicating acute myocardial infarction in the modern era with mechanical circulatory support: a single center observational study. Am J Cardiovasc Dis 6(1): 10-16.

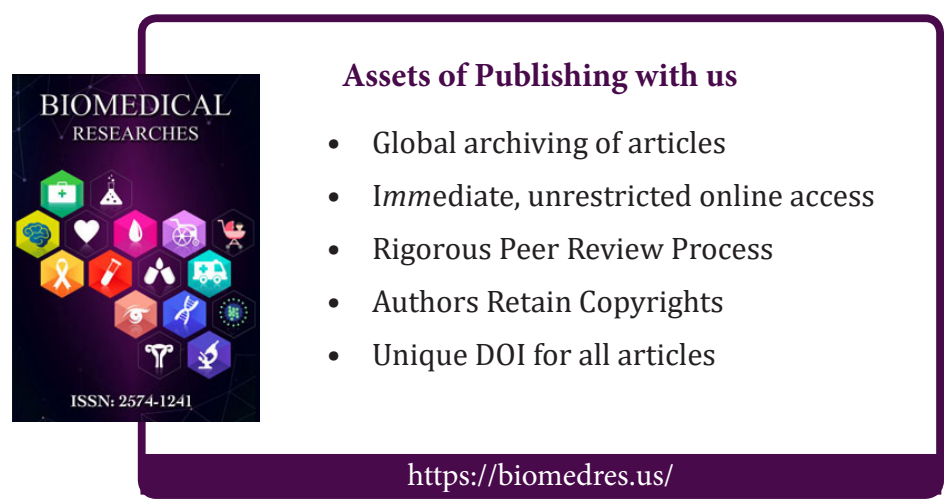

